\title{
Detectability of Stellar Companions by Imaging
}

\author{
WILLIAM D. HEACOX \& JOHN GATHRIGHT \\ Space Science Center, University of Hawaii, Hilo, HI 96720, USA
}

\section{EXTENDED ABSTRACT}

The increasing use of CCD's and IRCCD's to detect stellar companions, either by direct imaging or speckle techniques, raises the issue of the fraction of companions whose instantaneous, projected separations lie in the range corresponding to the geometric limits of the detector. The issue is of especial importance to recent surveys employing IRCCD detectors of relatively very small area (projected onto the sky). The distribution of observed separations reflects not only that of orbital semi-major axes, but also distributions of distances to binary systems, orbital eccentricities, tilt of orbital planes with respect to the plane of the sky, and of arguments of periastron. Proper inference of, e.g., binary multiplicity requires estimates of completeness that should incorporate contributions from all these factors.

We have statistically modeled this "visibility" problem to allow direct calculation of the distribution of observed, projected separations from an assumed distribution of semi-major axes amongst the primaries, in an observing program to image companion objects. We have explicitly assumed uniform distributions of orientations of orbital planes and of orbits within their planes, and of orbital eccentricities within the range [0,1]. This last assumption appears less certain than the first two, but is apparently compatible with the largest unbiased survey in the literature (Duquennoy \& Mayor 1991). The combined effects of distributions of orbital eccentricities and orientations are characterized by the function $\Gamma$ displayed below; to first order, these distributions cast fractions of secondaries both to closer and to further separations than would be the case with all-circular orbits seen face-on, and reduce the mean separation to 0.914 of the projected semi-major axis.

The full mathematical model takes the form of an integral equation with the assumed distribution of semi-major axes, $A(a)$, appearing in the integrand:

$$
S(s)=\frac{1}{N} \int_{z=s / 2}^{\infty} \frac{1}{z} \Gamma\left(\frac{s}{z}\right)\left[\sum_{i=1}^{N} d_{i} A\left(d_{i} z\right)\right] d z
$$

Here, $S(s)$ is the resulting distribution of observed, projected separations in arcseconds, $s ; d_{i}(i=1,2, \ldots, N)$ is the list of distances to the $N$ program stars in parsecs; and the geometric correction term $\Gamma$ is given by

$$
\Gamma(\chi)=\frac{2 \chi}{\pi^{2}} \int_{y=0}^{1} \frac{y}{1-y^{2}} \int_{\zeta=\chi}^{\min \left(2, \frac{x}{\sqrt{1-y^{2}}}\right)} \frac{\zeta \ln \left[\frac{1+\sqrt{\zeta(2-\zeta)}}{1-\zeta \mid}\right]}{\sqrt{\left(\frac{\chi^{2}}{1-y^{2}}-\zeta^{2}\right)\left(\zeta^{2}-\chi^{2}\right)}} d \zeta d y
$$

This model can be used in various ways to analyze the results of imaging surveys for stellar companions. If one is willing to assume an a priori distribution 
of companion semi-major axes, $A(a)$, the best estimate for the completeness of the survey will be

$$
C=\int_{x=0}^{\infty} S(x) D(x) d x
$$

where $D(x)$ is the probability that a separation of $x$ arcseconds will fall on the sensitive area of the detector (cf. Figure 5 of Skrutskie et al. 1989 for a graphical example). A more conservative approach involves the concept of a Detectability Function, defined as the probability that any randomly selected star in the program, with a putative companion of semi-major axis $a$, will have an instantaneous, projected separation that is detectable with the chosen detector; this is just the completeness statistic given above with the function $A(a)$ replaced by a Dirac delta function at the selected value of $a$. Such a statistic is free of any assumptions regarding the form of the (usually poorly known) $A(a)$, and makes explicit the likelihood that binaries of given semi-major axes can be detected in a given program.

In principle one can do much better, and infer the actual distribution of semi-major axes, $A(a)$, from the distribution of observed angular separations, $S(s)$, by inversion of the first equation above. But in practice such an inversion magnifies observational noise to the point that the results are often largely meaningless; cf. Craig \& Brown (1986) for an extensive discussion of the difficulties inherent in such inverse problems.

We have used our model to analyze a particularly important observation, the infrared survey for low-mass companions to field $M$ dwarfs of Skrutskie et al. (1989). We calculate a detectability function (for their sample of primary distances and detector geometry) that peaks sharply at approximately 0.2 for semi-major axes in the range of 20-30 A.U. Given their choice of assumed $A(a)$ (which peaks near 100 A.U.) we infer a completeness statistic of 0.053 ; in terms of their detection of one companion amongst 55 field primaries, we thus estimate the true multiplicity in their sample as approximately 0.34 , or about one-third. This is approximately twice the multiplicity the authors estimate from a less sophisticated analysis, a result which we interpret as demonstrative of the utility of statistical modelling of such data. We also argue that $a$ priori assumption of a distribution of semi-major axes deprives such completeness statistics of much meaning, and that the detectability function (as herein defined) is the most useful tool to analyze the results of surveys for companions that are insufficient in scope and quality as to allow the direct inference of $A(a)$; binary companions to $\mathrm{M}$ dwarfs with semi-major axes between approximately 20 and $30 \mathrm{~A}$.U. appear to be rare, but little else can be confidently concluded.

The full statistical model will be published elsewhere, as part of a program to statistically model most aspects of the detectability of stellar companions by various techniques.

\section{REFERENCES}

Craig, I.J.D. \& Brown, J.C. 1986, Inverse Problems in Astronomy, (Adam Hilger, Bristol)

Duquennoy, A. \& Mayor, M. 1991, $A \mathcal{E} A, 248,485$

Skrutskie, M.F., Forrest, W.J., \& Shure, M. 1989, $A J, 98,1409$ 